Convergences francophones 6.2 (2020): 83-94

http://mrujs.mtroyal.ca/index.php/cf/index

\title{
Former les enseignants du primaire à l'intégration des langues et cultures des Métis : enjeux et perspectives en contexte francophone minoritaire
}

Eva Lemaire

Université de l'Alberta

L'éducation est, pour Sinclair (What is Reconciliation?), la clé de la réconciliation entre les peuples autochtones et non autochtones. De fait, en réponse aux appels de la Commission de vérité et de réconciliation, le ministère de l'éducation albertain a entamé une réforme des programmes scolaires visant notamment une meilleure intercompréhension entre les peuples et une plus grande intégration des langues, cultures et perspectives autochtones à travers tout le curriculum. À compter de septembre 2019, les enseignants devront pouvoir être en mesure de mettre en œuvre cette orientation nouvelle. Alors que, de manière générale, se pose la question de la formation des enseignants et du manque de ressources pédagogiques adaptées, surtout en langue française, notre projet de recherche entend partir de la singularité du contexte francophone minoritaire albertain pour construire des ponts inédits entre communautés scolaires francophones et communautés métisses, prenant pour cela le michif comme point de convergence. Le michif, «langue officielle de la nation métisse (Atlas 24), est en effet une langue historiquement dérivée du français, dont l'exploration pourra donc sembler particulièrement pertinente dans des contextes scolaires où la langue de scolarisation est le français, comme c'est le cas dans les écoles francophones et dans les programmes d'immersion française de l'Ouest canadien. Dans cet article, nous commencerons par évoquer le contexte linguistique et éducatif lié à la recherche, avant de décrire le dispositif de recherche en cours, et partager ensuite quelques résultats préliminaires.

\section{Le michif : de quoi parle-t-on?}

Le Conseil National des Métis (Métis National Council, MNC), organe national de gouvernance des peuples Métis, définit les Métis comme les «descendants des Français engagés dans la traite des fourrures, et des femmes des Premières Nations («Michif» paragraphe 1) ${ }^{1}$. Bien que cette définition puisse paraitre simplificatrice, nous la mentionnons ici puisqu'elle émane de la voix politique des Métis du Canada. Comme le souligne Iseke, la définition de l'identité des Métis suscite en effet de nombreux débats et controverses sur lesquels nous ne nous arrêterons pas ici. L'auteure se réfère quant à elle à une définition selon laquelle est Métis « une personne qui s'auto-identifie comme Métis, ... qui a des ancêtres identifiés dans la communauté métisse historique et qui est reconnu par la Métis Nation » (94). Cette représentation de l'identité des Métis se rapproche par ailleurs de celle fournie dans l'Atlas des Peuples autochtones du Canada qui précise que:

le fait d'être Métis avec un grand "M" repose sur une définition politicoculturelle de l'identité métisse. Pour cette communauté, être Métis, ce n'est

\footnotetext{
${ }^{1}$ Nous traduisons.
} 
pas seulement avoir des ancêtres à la fois autochtones (en général, cris, saulteaux ou dénés) et européens ou eurocanadiens (le plus souvent, canadiens-français, écossais ou orcadiens) : c'est aussi se rattacher à un groupe de personnes qui se considèrent comme Métis et reconnaissent que leurs ancêtres ont pris la décision politique, fondée sur une histoire et une culture commune, de se dire "Métis". Pour les tenants de cette identité, il n'est pas seulement question de leur ascendance. S'il n'en tenait qu'à ça, presque toutes les Premières Nations du Canada, ainsi que les Canadiens français et les Acadiens, seraient aussi Métis, compte tenu du mélange génétique et culturel qui a eu dans les premiers temps de la colonie. (8)

La définition de ce qu'est le michif est tout aussi complexe. Différentes variations de michif coexistent en effet sur le territoire sur lequel les Métis ont fait vivre et évoluer leurs pratiques linguistiques au fil du temps et au gré des contacts avec les autres communautés linguistiques, qui les ont influencés de manière plus ou moins prégnante (Iseke 95). De manière générale, le michif est considéré comme une « langue mixte », à part entière (Bakker A Language, 160 ; Iseke 95; Rosen 613). Plusieurs linguistes se sont intéressés à décrire un michif empruntant principalement au français et au cri, avec influence d'autres langues autochtones telles que l'ojibwé, et avec une structure linguistique clairement établie. C'est à cette réalité linguistique que le MNC renvoie lorsqu'il définit le Michif sur son site web. C'est aussi ce que décrit Rosen: « la plupart des verbes sont issus du cri des Plaines alors que la plupart des noms et autres éléments nominaux tels que les déterminants, les chiffres, les adjectifs et les pronoms possessifs sont issus du français » (613). La phrase suivante, tirée de notre recherche avec une aînée métisse de la région de Lac La Biche (Alberta), Cécile Boucher Howse, est représentative de ce michif décrit par Rosen : " lii rassad ôhi sikasowak ». ${ }^{2}$ Dans cette phrase qui peut être traduite par «Ceci, ici, ça s'appelle des rassades (des perles)», « lii rassad » - les perles - sont en effet un déterminant et un nom dérivés du français, alors que l'adverbe « ôhi » (ici) et le verbe proviennent du cri.

Le linguiste Robert Papen a par ailleurs décrit une autre variante de michif, qu'il appelle le « Métis » (Quelques remarques 113) ou encore le « français michif» (Sontaient 201), et qu'il décrit comme un «parler français méconnu de l'Ouest Canadien » (Quelques remarques 113). Il en analyse, avec Bigot, quelques-unes des particularités parmi lesquelles une forme particulière d'imparfait telle que « sontaient » (113) que l'on retrouve dans notre corpus de recherche lorsque l'aînée avec laquelle nous avons travaillé affirme que « les sœurs sontaient méchantes » à l'école qu'elle fréquentait, enfant. L'Atlas des peuples autochtones du Canada fait également référence à ce «michif français » ou «français métis », qu'il décrit comme un « dialecte du français canadien ... difficilement compréhensible pour les francophones » (25).

2 «Rassade» est un terme de vieux français renvoyant aux petites perles de verre utilisées par Autochtones pour les ornementations. https://www.cnrtl.fr/definition/rassade. À noter également que l'orthographe du michif, langue orale, n'est pas fixée et que nous ne nous sommes pas arrêtée à une convention spécifique. 
Cet Atlas confirme aussi le fait rapporté par Iseke Barnes (93) selon qui le michif est aussi appelé « cri » par certains locuteurs. Dans ce cas de figure, le michif cri parlé comprend essentiellement du cri, la part du français étant très restreinte et limitée à quelques noms ici et là. L'influence de l'anglais, langue dominante en Alberta, est enfin également présente dans la langue michif. Iseke (95) rappelle ainsi que, pour Campbell (2), aînée et académique métisse reconnue, le dialecte michif parlé dans sa famille et sa région natale était nommé « village English », en écho au «mauvais français» (Brown, paragraphe "Structure Linguistique »; «Atlas », 25) qui a pu également qualifier dans le temps un michif à résonance plus francophone. L'article de l'Atlas des Peuples autochtones du Canada consacré au michif (24) distingue enfin le dialecte «nordique », parlé autour de Ile-à-la-Crosse, le « dialecte $\mathrm{N}$ », parlé par les Métis autour de Cumberland House et le « dialecte Y », parlé dans le sud de la Saskatchewan, à Lac La Biche en Alberta et dans les établissements métis albertains.

Cerner ce qu'est le michif est ainsi une tâche ardue. La multiplication des appellations utilisées par les locuteurs de michif renvoie à des pratiques hétérogènes de la langue selon les régions, mais aussi selon les individus. Comme l'indique Bakker (A language 160), la connaissance (équilibrée ou non) du français ou du cri par un locuteur peut avoir une influence sur la quantité de français et de cri qui sera utilisé dans le michif de la personne. Lors de nos discussions avec Cécile Boucher Howse, celle-ci admettra en effet que son michif varie selon les personnes avec qui elle interagit, le michif parlé par exemple avec nous, chercheure francophone native de la France, se teintant de davantage de français à notre contact. Cécile nous expliquera par ailleurs que ce michif, ancré davantage dans le français que dans le cri, était celui parlé par sa famille, implantée autour de la mission francophone Notre-Dame des Victoires.

Alors que Papen pose la question de savoir si la prolifération des termes et pratiques renvoyant aux Michifs constitue un « dédale sans issue " (La question 253), nous préférons quant à nous parler de continuum de pratiques linguistiques qui, sous l'appellation "michif», renvoie à ce qu'ont toujours été les Métis, des communautés métissées, ancrées dans divers lieux à travers le territoire, et qui n'ont eu de cesse de s'adapter, au contact des autres, au processus de colonisation et d'assimilation qui marque l'histoire canadienne. L'un des impacts majeurs de ce processus, qu'il est essentiel de préciser ici, est la perte du michif qui est devenu de nos jours une langue en voie de disparition, comme en atteste l'Atlas UNESCO des langues en danger dans le monde. Selon le recensement de Statistiques Canada de 2016, quelque 1170 personnes ont déclaré pouvoir tenir une conversation en michif, essentiellement en Saskatchewan. En Alberta, 145 personnes ont déclaré avoir une connaissance du michif. 80 ont le michif pour langue maternelle et 10 le parlent le plus souvent à la maison. Dans le cadre de notre projet de recherche, nous travaillons actuellement avec plusieurs aînés locuteurs de michif, nés en Alberta et pour qui le michif était la langue maternelle. Pour cet article, nous nous concentrerons sur la recherche entreprise avec Cécile Boucher Howse, grâce à qui nous avons pu créer une première trousse pédagogique bilingue français-michif que 
Convergences francophones 6.2 (2020): 83-94

http://mrujs.mtroyal.ca/index.php/cf/index

nous avons commencé à expérimenter auprès d'élèves et d'enseignants des écoles francophones et des programmes d'immersion française.

\section{Une trousse pédagogique français-michif pour intégrer langues et cultures des Métis dans les écoles de langue française}

En tant que didacticienne des langues et des cultures, nous avons élaboré une trousse d'éveil aux langues et cultures des Métis basée sur des ressources en michif. Ces ressources prennent la forme de vidéos, d'un livre numérique et de clips audios qui ont été déterminés en fonction des histoires de vie et savoir-faire que Cécile Boucher Howse était désireuse de partager. La trousse aborde trois principaux sujets : le perlage traditionnel métis que Cécile explique, ses souvenirs de l'école La Mission de Lac Labiche, ainsi que le récit d'une partie de pêche lorsqu'elle était adolescente. Ces thèmes permettent d'aborder des savoir-faire comme le perlage, le fumage du poisson, la trappe, ou encore des modes de vie traditionnels comme le fait de vivre des produits de la terre au contact du territoire; mais ils permettent aussi de mettre en avant une perspective métisse sur l'éducation reçue ou sur la manière dont modes de vie et langues ont évolué à travers le temps. La particularité de cette trousse est aussi qu'elle permet d'aborder des réalités locales, albertaines, quand les ressources qui existent par ailleurs tendent à être davantage ancrées en Saskatchewan et au Manitoba autour de l'institut Gabriel Dumont (à Régina) et du Louis Riel Institute (Winnipeg). De même, la littérature en michif produite par des auteurs Métis connus tels que Fleury, Flett ou encore Dorion permet certes d'entendre des histoires métisses, en langue originale, mais pas nécessairement les variantes de michif parlées en Alberta. Or, culture et identité autochtones sont fortement ancrées dans le territoire, comme en témoignent notamment les travaux de G.W Eades (4).

La trousse comprend, outre les ressources brutes, des plans de leçons et fiches d'activités "clés en main » qui permettent aux enseignants de la $4^{\mathrm{e}}$ à la $6^{\mathrm{e}}$ année d'exploiter les ressources en lien avec les programmes d'études ciblés. L'originalité de cette trousse pédagogique est également qu'elle a été créée avant tout pour un public d'élèves étudiant en langue française, et non en anglais.

Par ailleurs, les plans de leçons se veulent multidisciplinaires, en lien avec l'approche prônée en pédagogie autochtone notamment par Campeau, Goulet et Goulet ou encore Aluli-Meyer. Les activités prévues permettent ainsi de couvrir certains aspects du curriculum en études sociales, en arts, en sciences, mais aussi en français. En effet, dans la mesure où le point de départ est bien la langue et le fait que le michif peut être vu comme une langue affiliée au français, notre approche s'inscrit d'abord dans la démarche didactique dite de l'éveil aux langues. Cette démarche a démontré son potentiel pour le développement des compétences plurilingues et pluriculturelles des élèves, ainsi que pour le développement de leurs attitudes citoyennes, comme indiqué notamment dans les travaux de Candelier. Cette démarche d'apprentissage, qui s'adresse à tout élève indépendamment de son niveau ou de ses bagages linguistiques, aborde de front plusieurs langues ou variétés de langues, qu'elles soient connues ou pas des élèves. Elle invite donc 
enseignants et apprenants, dotés de leur seule curiosité et d'un esprit d'analyse, à se plonger dans l'univers de la diversité linguistique et culturelle. Les élèves mènent alors l'enquête, testent des hypothèses, pour explorer les langues. Concrètement, dans le dispositif que l'on propose, les élèves devront par exemple proposer une explication pour justifier l'absence de l'accord de l'adjectif dans le segment en michif « lii fleurs sont pchi oota $»^{3}$ quand l'adjectif s'accorde en genre et en nombre dans cet autre segment: « lii pchites fleurs ». Dans l'éveil aux langues, l'enseignant lui-même peut n'avoir des connaissances que très partielles des langues impliquées, la démarche reposant, comme l'indique Dagenais dans ses travaux, avant tout sur une démarche de réflexivité, de co-construction des savoirs et d'ouverture linguistique.

Pour autant, la trousse d'éveil aux langues ne se limite guère à cette approche linguistique, la langue étant un vecteur pour aller à la découverte d'histoires et de savoir-faire traditionnels racontés en langue.

\section{Le dispositif de recherche-action-formation}

Pendant une année académique, quatre enseignants ont accepté d'expérimenter la trousse pédagogique en question. Trois d'entre eux étaient des enseignants d'écoles francophones de la région d'Edmonton et de Red Deer, quand le dernier enseignant œuvrait en immersion, dans le nord de la province. Le premier enseignant enseignait au niveau de la $4^{\mathrm{e}}$ année au moment de l'expérimentation, le second en $5^{\mathrm{e}}$ année, le troisième en $6^{\mathrm{e}}$ année et le quatrième avait un niveau double $5 / 6^{\mathrm{e}}$ année. Les enseignants avaient accès à la même trousse, qui propose des pistes d'adaptations pédagogiques selon le niveau et les centres d'intérêt de la classe. Chaque enseignant avait le choix d'utiliser une ou plusieurs ressources en michif, de modifier ou compléter plans de leçons et feuilles d'activités, et de planifier les activités pendant l'année à sa convenance. Tous les enseignants ont utilisé au minimum les trois ressources de base (deux vidéos et le livre numérique), ont fait des modifications mineures à la trousse pédagogique pour qu'elle reflète leurs besoins et ont réparti les activités tout au long de l'année scolaire. À noter que les enseignants avaient accès en tout temps à une plateforme intranet sur laquelle se trouvait hébergées la trousse ainsi que quelques ressources d'autoformation reliées à l'utilisation de la trousse. Le dispositif se veut en effet un dispositif de rechercheaction-formation, tel qu'envisagé par Charlier. Ce dispositif doit permettre aux enseignants volontaires de se former au contenu et à l'approche en question afin de pouvoir l'enseigner de manière la plus autonome possible. Dans le cadre de cette recherche collaborative, nous étions disponible en tout temps pour répondre aux questions des enseignants participant et les accompagner dans la mise en œuvre de la trousse. Nous étions ainsi présente pour les sessions où la trousse a été utilisée dans les classes.

${ }^{3}$ Il n'existe pas d'orthographe du michif qui soit standardisé («Atlas» 25) et qui fédère les différents dialectes. Nous avons ici opté pour une orthographe inspirée du Michif Dictionary de Norman Fleury. 
D'un point de vue méthodologique, les données dont nous disposons sont issues d'un devis mixte et sont constituées de :

- notes issues de l'observation participante dans une démarche « d'ethnographie de la classe » (Moore et Sabatier 8), alors que nous étions présente en classe pour observer et épauler les enseignants dispensant le contenu de la trousse à leurs élèves. Ces notes incluent les discussions de débriefing menées avec les enseignants à la fin de chaque session.

- un post et un pré-test auprès des élèves permettant d'analyser l'évolution de leurs connaissances et représentations par rapport aux Métis et au michif

- un entretien initial et final avec chaque enseignant

- un entretien final avec les élèves d'une des quatre classes

- des copies ou photographies du matériel pédagogique supplémentaire créé par les enseignants ainsi que des travaux réalisés par les élèves

L'analyse thématique de données, encore en cours, doit permettre d'analyser dans quelle mesure l'approche et le matériel s'avèrent pertinents pour enseigner le curriculum en y intégrant une perspective autochtone, tel qu'exigé par le nouveau standard de qualité de l'enseignement en Alberta. Il est question aussi d'évaluer, à travers ce processus, le besoin de formation des enseignants en écoles francophones et programmes d'immersion pour ce qui est de l'intégration des perspectives autochtones dans leurs pratiques professionnelles, et de proposer des pistes de solutions quant à la manière d'y répondre.

Il s'agit également de déterminer en quoi l'éveil au michif peut entrer en résonance avec le vécu des élèves en contexte scolaire francophone et amener un regard renouvelé sur les politiques linguistiques et éducatives, ainsi que sur les pratiques langagières en contexte minoritaire. Enfin, avec cette recherche, nous souhaitons évaluer comment un tel dispositif peut permettre d'établir de nouvelles connexions entre communautés scolaires francophones et communautés autochtones, dans une logique de réconciliation.

\section{Quelques résultats préliminaires}

\section{Une trousse adaptée à l'enseignement du curriculum}

De manière unanime, selon les enseignants, la trousse a permis d'intégrer langues, cultures et perspectives métisses au curriculum, se greffant donc au programme d'études, sans avoir à se surajouter sur un programme déjà chargé. Les enseignants se sont en effet servis de la trousse pour étudier les études sociales (en lien avec le programme d'études sur les Métis principalement), les arts (la représentation de la nature, la confection en 3D...), les sciences (les écosystèmes, les forêts), et le français (les stratégies de compréhension orale, le schéma narratif, la compréhension des idées implicites, etc.). Selon deux enseignantes, la trousse leur a donné de nouvelles idées pour travailler certains résultats d'apprentissages qu'elles avaient tendance à occulter, comme l'analyse de la phrase ou la critique d'art. Un enseignant précisera aussi en entretien que la trousse l'a invité à explorer davantage l'intérêt de la pédagogie expérientielle et de la pédagogie de la terre, une 
pédagogie autochtone dont la pertinence est analysée notamment par Wildcat et al. Les élèves ont, de fait, particulièrement apprécié d'aller en forêt identifier les arbres discutés en classe, avant de collecter des artefacts servant à la réalisation du perlage métis.

D'après tous les enseignants, les élèves ont montré une motivation au moins égale à l'habitude, voire supérieure dans trois des quatre classes, pour ce projet d'une année sur l'éveil au michif. Tous les enseignants estiment que leurs élèves ont démontré une plus grande compréhension des réalités autochtones, avec une perception désormais moins passéiste des Métis. La majorité des élèves ont, quant à eux, indiqué un désir d'en apprendre plus sur les Métis, et ont signifié avoir particulièrement aimé acquérir des connaissances sur les Métis à travers des projets d'art.

\section{Des enseignants rassurés sur leur capacité à enseigner les perspectives}

De façon univoque, les enseignants ont déclaré en entretien final avoir beaucoup appris par le biais de la trousse et de l'accompagnement fourni. Tous avaient reconnu en début d'année que l'enseignement des perspectives autochtones était essentiel, surtout avec l'arrivée du nouveau standard d'enseignement, mais tous admettaient aussi avoir des lacunes à combler au niveau de leurs connaissances; la plupart des enseignants avouant qu'ils se cantonnaient habituellement au manuel, de peur de faire erreur. Pour tous les enseignants, la trousse a permis de renouveler leur approche pour y apporter une dimension plus relationnelle, plus authentique et moins tournée vers le passé. Le projet a également permis aux enseignants de s'interroger sur des représentations inexactes, celles qui amènent par exemple à affirmer à tort que les Métis sont à moitié autochtones ou qu'est Métis tout individu qui a un parent francophone et un parent des Premières Nations. Une des enseignantes remarquera par exemple pour la première fois que la définition des Métis fournie par le manuel scolaire utilisé est inexacte. Comme le note enfin un des enseignants interrogés en entretien final, le projet aura fait apparaître le besoin de continuer à se former davantage, tant il est vrai que les programmes scolaires tendent à ne parler des Métis qu'à l'époque des voyageurs et de la résistance menée par Louis Riel, laissant tout un pan de l'histoire et de la société contemporaine vacant.

\section{Une résonance particulière en milieu francophone et immersif}

Interrogés sur la question, tous les enseignants se sont dits agréablement surpris de constater la curiosité des élèves envers le michif, une langue avec laquelle ils ne s'attendaient pas que les élèves fassent autant de liens. Invités à reconnaître les mots français présents dans le michif, à deviner le sens des mots et à déchiffrer la logique intrinsèque de la langue, les élèves se sont appuyés sur leurs connaissances en français pour aller à la découverte d'une langue en voie d'extinction qui leur était inconnue.

Une des enseignantes, la seule à avoir en fait choisi de travailler la grammaire française à partir des plans de leçons disponibles, soulignera en entretien que la 
démarche de l'éveil au michif permet aux élèves de faire des hypothèses avec la langue, de se tromper en toute sécurité et de poser des questions quand, la plupart du temps, l'analyse grammaticale de la phrase est un aspect du programme soigneusement évité à cause de sa trop grande complexité. Dans ce cas spécifique, la démarche de l'éveil aux langues semble avoir atteint son but.

Un autre aspect que nous voulions aborder par le biais de ce projet ancré en didactique du plurilinguisme est la pratique des langues en milieu minoritaire. En effet, les recherches tendent à montrer qu'en contexte scolaire francophone minoritaire, l'école « joue un rôle de production et de reproduction de la langue et de la culture françaises » (Gérin-Lajoie 2) au détriment d'une valorisation des répertoires plurilingues. Dans le cadre de ce projet, nous avons pu observer des discussions émerger sur les pratiques bi ou plurilingues des élèves, notamment sur les diverses raisons expliquant l'alternance codique en contexte plurilingue. Plusieurs élèves ont ainsi pu valoriser leur bagage plurilingue, les enseignants pouvant en découvrir l'ampleur. On soulignera aussi à cette occasion la fierté évidente d'un élève à parler le créole et à faire des parallèles avec la langue mixte qu'est le michif, comme le mentionne son enseignante en entretien final. Les élèves, dans deux des classes concernées, auront aussi eu l'occasion d'une discussion sur l'impact des politiques linguistiques et éducatives pour favoriser le maintien des langues, comparant à cet égard les politiques favorables au français, langue officielle au Canada, et les politiques de soutien à la revitalisation des langues autochtones en lien avec les revendications des Premières Nations, des Métis et des Inuits (PNMI).

Aux dires des enseignants, la démarche de l'éveil au michif ouvre donc une nouvelle porte à la discussion sur les pratiques langagières en milieu minoritaire.

\section{Des connexions nouvelles avec la communauté}

Dans chacune des écoles, le projet a débouché sur le désir de créer des liens avec les communautés autochtones, le plus souvent via la création de liens directs avec Cécile, personnage au centre du dispositif. Dans une des quatre écoles, le désir de correspondre avec Cécile a été exprimé, mais n'a pas donné suite, faute de temps. Dans une autre école, l'enseignante a souhaité inviter l'aînée à l'occasion de la journée nationale des peuples autochtones, le 21 juin, mais cela n'a pu se faire pour des questions essentiellement logistiques. Les élèves ont par contre eu la possibilité de poser des questions précises à l'illustratrice métisse qui a illustré le livre numérique; questions auxquelles ils ont pu avoir des réponses par notre entremise. Les élèves d'immersion du nord de la province ont quant à eux écrit des messages à Cécile Boucher Howse. Bien qu'il puisse y avoir là un biais de « désirabilité sociale » (Fisher 303), les élèves écrivant ce qu'ils pensent être attendu de l'enseignant et du chercheur, les messages rédigés témoignaient de leur appréciation quant à la culture, de leur appréciation quant à la relation nouée indirectement via la vidéo et la correspondance; certains messages témoignant aussi 
d'une sensibilité accrue au vécu des survivants des écoles résidentielles. On citera ici les messages suivants, adressés à Cécile: ${ }^{4}$

« J'adore tes histoires, merci pour les partager avec nous. Moi, je suis Métis et je ne sais comment parler le michif. Je pense que c'est incroyable tes histoires. Alors merci. »

«Chère Cécile, j'aime l'histoire que tu as raconté. Tu es brave à raconter cette histoire-là ».

«Chère Cécile. Je suis désolée à toi. C'est injuste ce que tu as dû faire et alors je vais faire sûr de porter de l'orange pour toi ».

Cécile répondra aux élèves par le biais d'une vidéo personnalisée, ce que la plupart des élèves interrogés en entretien final souligneront avoir beaucoup apprécié. Enfin, il est à noter que l'enseignant de cette classe, suite au projet, a décidé de nouer des contacts avec la section locale Métis de sa région, local dont il ignorait l'existence au début du projet.

Enfin, une des classes participant au dispositif a choisi d'écrire un texte argumentatif, texte à étudier au programme, pour écrire une lettre à la directrice de l'école et réclamer une cérémonie de lever de drapeaux à l'occasion de la journée nationale des peuples autochtones. Le drapeau métis et le drapeau du traité 6 trônent désormais dans l'école. À notre connaissance, ce fut la première fois que ces drapeaux étaient hissés à cette occasion et en cérémonie, sous la guidance spirituelle d'un gardien du savoir, dans une des écoles du conseil scolaire francophone concerné.

\section{Conclusion}

Tous les enseignants qui ont participé au dispositif se sont dits ravis d'avoir participé à l'expérience tant cela répondait à un besoin de formation. Chacun se sent désormais davantage outillé pour poursuivre son cheminement et intégrer davantage langues, cultures et perspectives autochtones dans leurs pratiques professionnelles. Pour tous, la dimension humaine et relationnelle, à travers la rencontre avec Cécile, fut l'une des clés du succès. Si quelques enseignants ont ainsi saisi l'opportunité de ce projet de recherche-action-formation pour nouer des liens avec la communauté locale autochtone, on soulignera en conclusion l'importance pour les conseils scolaires francophones de développer ces liens avec les aînés, gardiens du savoir et autres leaders communautaires pour permettre à un véritable processus de réconciliation de se mettre en œuvre, à travers l'établissement d'un dialogue de qualité, à long terme. Contrairement à de nombreux conseils scolaires anglophones de la province qui travaillent déjà avec des consultants, des aînés et organismes autochtones, et qui forment pour chaque école des enseignants spécialisés référents («lead teachers»), les écoles francophones apparaissent démunies quand notre projet démontre pourtant un réel appétit des élèves et des enseignants en la matière.

\footnotetext{
${ }^{4}$ Nous avons ici conservé les messages dans leur forme originale.
} 


\section{Bibliographie}

Alberta Education. Norme de qualité pour l'enseignement, 2018, https://education.alberta.ca/media/3576234/nqe-norme-enseignement.pdf. Entrée consultée 9 juil. 2019.

Bakker, Peter. A Language of Our Own: The Genesis of Michif, the Mixed CreeFrench Language of the Canadian Métis. Oxford University Press, 1997.

---. "What is Michif, and What is Called Michif?" La lawng: Michif peekishkwewin: The heritage language of the Canadian Métis, édité par L. Barkwell et N. Fleury, Pemmican, 2004, pp. 5-7.

Barkwell, Lawrence et Norman Fleury. La lawng: Michif Peekishkwewin : the heritage language of the Canadian Metis: Manitoba Metis Federation Michif Language Program. Pemmican, 2004.

Brown, Jennifer, S.H. “Mitchif”. L'Encyclopédie canadienne, 3 août 2018, https://www12.statcan.gc.ca/census-recensement/2016/dp-pd/dvvd/lang/index-eng.cfm Entrée consultée 9 juil. 2019.

Canadian Geographic. Atlas des Peuples autochtones du Canada. Les Métis. Ottawa, 2018

Campeau, Diane. Pédagogie autochtone. Présentation donnée au Campus SaintJean, University of Alberta, 23 mars 2017.

Campbell, Maria. Stories of the Road Allowance People. Theytus, 1995.

Candelier, Michel. « Approches plurielles, didactiques du plurilinguisme: le même et l'autre ». Les Cahiers de l'Acedle, vol. 5, no 1, 2008, pp. 65-90.

Candelier, M. et al. Le CARAP : un cadre de référence pour les approches plurielles des langues et des cultures : compétences et ressources. Conseil de l'Europe, 2012, https://www.ecml.at/Portals/1/documents/ECMLresources/CARAP-FR.pdf?ver=2018-03-20-120658-740

Charlier, Bernadette. " Parcours de recherche-action-formation ». Revue des sciences de l'éducation, vol. 31, $\mathrm{n}^{\circ} 2,2005$, pp. 259-272.

Commission de Vérité et de réconciliation du Canada. Honorer la vérité, réconcilier pour l'avenir. Sommaire du rapport final, 2015, www.trc.ca

Dagenais, Diane, et al. "Collaboration and co-construction of knowledge during language awareness in Canadian Elementary School". Language Awereness, vol. 17, no 2, 2008, pp. 139-155.

Dorion, Leah. My first Métis Lobstick. Gabriel Dumont Institute, 2014.

---. The Giving Tree: A Retelling of a Traditional Metis Story. Gabriel Dumont Institute, 2009.

---. Relatives with Roots. Gabriel Dumont Institute, 2011.

Eades, Gwilym Lucas. Maps and Memes: Redrawing Culture, Place, and Identity in Indigenous Communities. McGill Queen's University Press, 2015.

Fisher, Robert J. "Social Desirability Bias and the Validity of Indirect Questioning". Journal of Consumer Research, vol. 20, n 2, 1993, pp. 303315.

Flett, Julie. Owls See Clearly At Night (Lii Yiiboo Nayaapiwak Lii Swer): A Michif Alphabet (L'Alfabet Di Michif). Strong Nations, 2010. 
Fleury, N. Mitchif Dictionnary. Gabriel Dumont Institute, 2013.

Gérin-Lajoie, Diane. « Identité bilingue et jeunes en milieu francophone minoritaire : un phénomène complexe », Le Réseau de recherche sur les nouvelles approches de l'éducation permanente, https://nall.oise.utoronto.ca/res/62DianeLerinLajoie.pdf. Entrée consultée 9 Juil. 2019.

Goulet, Linda M. et Keith N. Goulet. Teaching each other. Nehinuw Concepts \& Indigenous Pedagogies. UBC Press, 2014.

Iseke, Judy. "Negotiating Métis culture in Michif: Disrupting Indigenous language shift”. Decolonization: Indigeneity, Education \& Society, vol. 2, nº. 2, 2013, pp. 92-116.

Métis National Council. "Michif”, MNC, http://albertametis.com/culture/michif/. Entrée consultée 11 Juil. 2019.

Moore, Danièle et Cécile Sabatier. Une semaine en classe en immersion française au Canada. PUG, 2012.

Moseley, Christopher. "Atlas des langues en danger dans le monde », UNESCO, 2010, www.unesco.org/culture/languages-atlas/fr/atlasmap.html. Entrée consultée 11 Juil. 2019.

Papen, Robert A. «La question des langues des Mitchifs : un dédale sans issue ?». Histoires et identités métisses : hommage à Gabriel Dumont, édité par Denis Gagnon, Denis Combet et Lise Gaboury-Diallo, Presses universitaires de Saint-Boniface, 2009, p. 253-276.

---. « La diversité des parlers français de l'Ouest canadien : mythe ou réalité ? » Cahiers franco-canadiens de l'Ouest, vol. 16, $\mathrm{n}^{\text {os }} 1-2,2004 \mathrm{a}, \mathrm{pp} .13-52$.

---. « Les parlers français de l'Ouest canadien ». Cahiers franco-canadiens de l'Ouest, vol. 16, $\mathrm{n}^{\text {os }} 1-2,2004 \mathrm{~b}, \mathrm{pp}$. 1-9.

---. «"Les troub' ": analyse linguistique d'un texte oral en français des Métis ». Cahiers franco-canadiens de l'Ouest, vol. 14, nos 1-2, 2002, pp. 61-88.

---. «Quelques remarques sur un parler français méconnu de 1'Ouest canadien : le métis ». Revue québécoise de linguistique, vol. 14, n 1, 1984, pp. 113-139.

Papen, Robert A. et Davy Bigot. « Sontaient, ontvaient, et fontsaient en français michif : variation et systématicité ». Vues sur les français d'ici, édité par Carmen Leblanc, et al., Presses de l'Université Laval, 2010, pp. 201-225.

Rosen, Nicole. "French-Algonquian Interaction in Canada: A Michif case study". Clinical Linguistics \& Phonetics, vol. 22, no8, 2008, pp. 610-624.

Sinclair, Murray. "What is Reconciliation", TRC, YouTube, téléchargé par Andy Burns, 2 décembre 2016, https://binged.it/2MJ3RiZ

Statistiques Canada. "The Aboriginal languages of First Nations people, Métis and Inuit. Census in brief. 25 oct. 2017, https://www12.statcan.gc.ca/censusrecensement/2016/as-sa/98-200-x/2016022/98-200-x2016022-eng.cfm. Entrée consultée 9 juil. 2019.

Statistiques Canada. Janvier 194, IRI [Census subdivision], Alberta and Alberta [Province] (table). Census Profile. 2016 Census. Statistics Canada Catalogue no. 98-316-X2016001, 29 nov. 2017, Entrée consultée 11 Juil. 2019 
https://www12.statcan.gc.ca/census-recensement/2016/dp$\mathrm{pd} /$ prof/index.cfm?Lang=E

Wildcat, Matthew, et al. "Learning from the land: Indigenous land based pedagogy and decolonization". Decolonization, Indigeneity, Education and Society, vol 3, n ${ }^{\circ} 3,2014, \mathrm{pp} .1-15$. 